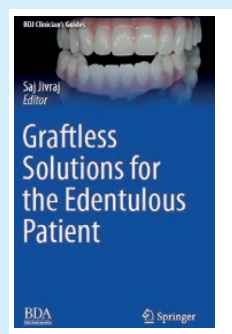

\section{BDJ CLINICIAN'S GUIDES: GRAFTLESS SOLUTIONS FOR THE EDENTULOUS PATIENT}

Saj Jivraj

Springer; 2018

Price $\mathrm{f} 85.24$ (non-member price $\mathrm{f} 109.99$ ) pp. 434

ISBN: 9783319658575

This book is a detailed clinical guide for planning and placing implants in edentulous patients. It provides clinicians who place implants with a framework to base their investigation and plan implant placement.

The book has an excellent list of contributors who share their wealth of knowledge and provide excellent clinical guidance and suggestions on how to plan implant treatment in edentulous patients.

As a dentist beginning his implant journey, I really enjoyed that the book incorporated digital dentistry into its content, covering CADCAM laboratory manufacture, covering materials such as

The $B D J$, in association with the BDA, have published the first in a new series of books: the BDJ Clinician's Guides. This new series will build on the trusted BDJ Clinical Guides to create books that update and inform the busy clinician on key topics across all fields of dentistry. Each volume is authored by recognised experts, is superbly illustrated and provides concise, highly practical guidance and solutions. The first book in the series is Graftless solutions for the edentulous patient, so we spoke to the editor, Dr Saj Jivraj, to find out more about it.

\section{Why should people read this book?}

This book is designed to present a systematic and practical approach to restoring the edentulous and soon to be edentulous patient with fixed implant-supported restorations. The data shows that the number of edentulous patients is increasing and clinicians will need to be familiar with the treatment strategies in treating these types of patients. This text is comprehensive in that it addresses protocols for diagnosis, treatment planning, surgery, restoration and maintenance. It also covers laboratory techniques so that the end result is aesthetically acceptable and biomechanically sound.

This text is written in collaboration with a host of well-respected clinicians, authors and teachers, who are leaders in their field. These clinicians have years of experience in this field and are well published and provide this treatment in their daily private practices.

\section{Who is it aimed at?}

This book is written for general dentists, surgical specialists, prosthodontists, laboratory technicians, hygienists and dental assistants. Representatives from implant companies could also benefit from the detailed knowledge about this treatment protocol.

\section{Did you enjoy writing it?}

Full mouth restoration using dental implants is my passion.

Collaborating with world renown clinicans, authors and teachers
PEEK, and highlighting the considerations which much be given when designing these types of frameworks.

The book covers the numerous different approaches that can be taken when planning implants for the edentulous patient such as guided implant surgery, graftless surgery techniques and immediate loading of these cases. The laboratory and prosthetic stages are well documented and rationale of a particular choice of material is given great consideration.

The clinical patient presentations in chapter 17 are very well documented and presented, with good case commentary, making it easy for implant dentists at any stage of their career to follow.

In summary, this is an excellent clinical guide, covering a range of emerging new techniques in implant dentistry such as graftless procedures. The clinical guide is very accessible in my opinion and I feel it would be beneficial to all implant dentists at various stages of their career, and great credit must be given to the authors for the excellent structure and presentation of the clinical guide.

Jay Joshi

Available to buy from the BDA shop: https://shop.bda.org

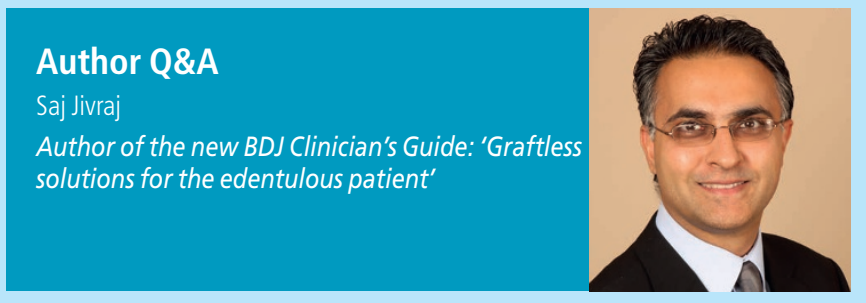

was very rewarding. So yes I very much enjoyed writing this text. I'm already looking forward to the second edition.

\section{What made you write it?}

I felt the dental profession did not have a comprehensive text in treating this type of patient. I began treating patients using this protocol almost 13 years ago. When I started there was nothing out there to really explain the techniques and the protocols for success. I thought it would be great to collaborate with some of the world's best clinicians/authors and bring their experience to the forefront so others may obtain this information from one source

\section{Any advice for future authors of BDJ Clinician's Guides?}

Be organised, be prepared and be ready to roll your sleeves up. It's hard work but when it all comes to fruition it's a great feeling

\section{What do you do in spare time?}

With writing, teaching and private practice I spend a lot of time away from my family. So all the spare time I have is spent with the people who matter most - my wife, Dilaz, and two children, Sara and Zain. We love to travel and experience different cultures.

\section{What other dentistry books would you highly recommend?}

One comes to mind and it happens to be part of a BDJ clinical series: Treatment planning in implant dentistry by S. Jivraj and W. W. L. Chee. We are ready for the second edition so watch this space. 